\title{
Disfagia en el paciente neurocrítico: ¿Que debemos saber los neurocirujanos?
}

Karen Muñoz Báez¹, Andrés Felipe Munive-Beltrán1, Mónica Patricia Herrera-Martínez¹, Loraine Quintana-Pájaro', Yancarlos Ramos-Villegas ${ }^{1}$, Alfonso Pacheco-Hernández ${ }^{2}$, Luis Rafael Moscote-Salazar ${ }^{2}$

1 Universidad de Cartagena, Cartagena de Indias, Colombia. Centro de Investigaciones Biomédicas (CIB), Facultad de Medicina, Universidad de Cartagena, Cartagena Colombia.

2 Médico. Especialista en Neurocirugía. Facultad de Medicina, Universidad de Cartagena. Cartagena de Indias, Bolívar. Centro de Investigaciones Biomédicas (CIB), Facultad de Medicina, Universidad de Cartagena, Cartagena Colombia.

Rev. Chil. Neurocirugía 45: 140-143, 2019

\section{Resumen}

Algunos de los tratamientos usados a la hora de tratar al paciente crítico, como el uso del tubo endotraqueal, pueden llevar a complicaciones como la disfagia, definida como la dificultad o incapacidad para deglutir, secundaria a la disfunción en cualquiera de las cuatro fases de la deglución (preparación oral, propulsión oral, faríngea o esofágica). Su aparición y complicaciones se relacionan de manera directamente proporcional al tiempo de intubación y estadio en el hospital. Esta, tiene un impacto negativo en la calidad de vida del paciente, ya que repercute claramente en su estilo de vida, llevándolo a modificar su dieta, pérdida del apetito y malnutrición, entre otros. Además, puede ocasionar complicaciones como la aspiración o perforación, neumonía, deshidratación y falla respiratoria. No se ha determinado un tratamiento puntual a la hora de tratar la disfagia, sin embargo, se ha demostrado que el uso de ciertas maniobras puede mejorar el riesgo de aparición de complicaciones y la calidad de vida del paciente como la modificación de la textura de la dieta, cambios posturales, maniobras compensatorias, ejercicios terapéuticos y estimulación nerviosa.

Palabras clave: Disfagia, desórdenes en la deglución, paciente crítico, cuidado crítico, unidad de cuidados intensivos, fisiopatología de la deglución.

\begin{abstract}
Some of the treatments used to treat critical patients, such as endotracheal tube use, can lead to complications such as dysphagia, defined as difficulty or inability to swallow, secondary to dysfunction in any of the four phases of the procedure. swallowing (oral preparation, oral, pharyngeal or esophageal propulsion). Its appearance and complications are related in a manner directly proportional to the time of intubation and stage in the hospital. This, has a negative impact on the quality of life of the patient, as it clearly affects their lifestyle, leading to change their diet, loss of appetite and malnutrition, among others. In addition, it can cause complications such as aspiration or perforation, pneumonia, dehydration and respiratory failure. No specific treatment has been determined when treating dysphagia, however, it has been shown that the use of certain maneuvers can improve the risk of complications and the quality of life of the patient such as the modification of the texture of the patient, diet, postural changes, compensatory maneuvers, therapeutic exercises and nerve stimulation.
\end{abstract}

Key words: Dysphagia, deglutition disorders, critial patient, critical care, intensive care unit, deglutition pathophysiology. 


\section{Introducción}

Los pacientes admitidos en las unidades de cuidado crítico, independientemente del motivo de ingreso, suelen llegar con diversas comorbilidades, puntuaciones bajas en la escala de Glasgow y altos riesgos para su salud. Por el riesgo de falla ventilatoria, se les brinda soporte ventilatorio por medio de intubación orotraqueal ${ }^{1,2}$, resultando en diversas complicaciones ${ }^{3}$, de las cuales abordaremos en esta revisión la disfagia o trastornos de la deglución. La disfagia, definida como la incapacidad para tragar, es una condición común dentro de los pacientes en la Unidad de Cuidados Intensivos (UCI), con una incidencia del $20 \%$ en los pacientes con intubación orotraqueal ${ }^{3,4}$. Puede estar mediada por mecanismos mecánicos, disfunción neuromuscular de los músculos de la deglución en neuromiopatías, sensibilidad orofaríngea o laríngea y el reflujo gastroesofágico ${ }^{2,3,5}$. Los trastornos de la deglución poseen signos, como dolor y tos al tragar, ausencia del reflejo nauseoso e incluso disfonía, y pueden conducir a complicaciones mayores, como la aspiración, penetración, dolor y molestias al deglutir, deshidratación, infecciones respiratorias y falla respiratoria, entre otras ${ }^{2,4,6}$. Presentamos una sencilla revisión sobre disfagia y los aspectos relevantes de esta condición para contribuir al conocimiento de los neurocirujanos.

\section{Epidemiología}

La disfagia, definida como la dificultad o incapacidad para deglutir ${ }^{3,4}$, puede originarse a partir de diferentes etiologías. En esta revisión se abordará la disfagia neurogénica (como resultado de mecanismos propios del tubo endotraqueal, lesión de mucosa, entre otros, y mecanismos cognitivos que induzcan al detrimento del reflejo de deglución ${ }^{4}$, por ser una de las condiciones que frecuentemente se observa en pacientes admitidos en la $\mathrm{UCl}^{2}$. Ésta representa tres-cuartos de las etiologías de disfagia y su incidencia está estimada en rangos desde el $3 \%$ hasta el $83 \%^{1,3,5,7,8}$, donde al menos el $20 \%$ de pacientes entubados presentan posteriormente alguna implicación en su deglución ${ }^{9}$, acuñándose esta variación a la falta de consensos universales acerca de criterios diagnósticos a emplear, instrumen- tos para evaluación, diferencias entre las cohortes poblacionales y tiempos de evaluación de disfagia ${ }^{6,9}$.

El tamizaje y seguimiento ejercen gran impacto en el pronóstico del paciente, su calidad de vida, las complicaciones y los costos al Sistema de Salud ${ }^{9,10}$. Según la Asociación Americana de Ataque, el Ataque Cerebrovascular es la causa principal de disfagia en un $42 \%$ $67 \%$ dentro de los primeros 3 días $^{9}$, explicándose por la disminución del estado de consciencia, o por la intubación orotraqueal en pacientes sometidos a neurocirugía.

\section{Factores predisponentes}

La disfagia ocurre principalmente en pacientes de la unidad de cuidado crítico, sin embargo, no es exclusivo y puede producirse por disrupción neurológica o estructural en cabeza y cuello, intervenciones terapeuticas, patologías del paciente y factores no modificables como la edad ${ }^{3,6}$. Dentro de los principales tenemos: Ataque cerebrovascular, Cáncer en cabeza y cuello, cirugías de espina cervical, traqueostomía, múltiples intubaciones, tiempo de permanencia en $\mathrm{UCl}$ aumentado, falla cardíaca congestiva, aumento del tiempo operatorio, ecocardiografía transesofágica perioperatoria, sepsis y ventilación mecánica (donde cada día aumenta el rieso de disfagia en un $25 \%)^{3,4,9,11}$. Los pacientes con problema para deglutir que comen o beben pueden aspirar y seguidamente desarrollar falla respiratoria aguda e incrementar el riesgo de neumonía hasta 11 veces $^{2,8}$. Además, existen otras complicaciones como la deshidratación, malnutrición, broncoaspiración ${ }^{4}$.

\section{Fisiopatología de la disfagia en el paciente neurocrítico}

La capacidad para tragar es resultado de una organización intrincadamente cronometrada de eventos que involucran alrededor de 30 grupos musculares y dos centros cerebrales ${ }^{2}$ ). Este proceso se divide en 4 fases:

- Preparación oral: Consiste en la preparación de sólidos y líquidos para su tránsito seguro, comprende la masticación, un proceso de control voluntario. Son necesarios la saliva y el sello entre el paladar y la lengua, esto último impide el paso del bolo hacia la vía aérea.

- Propulsión oral: Se origina por control voluntario, produciendo una serie de contracciones en la lengua, piso de la boca y paladar blando, impulsando el contenido bucal hacia la parte posterior de la cavidad oral e introduciéndolo en la faringe.

- Faríngea: Inicia con la entrada del contenido bucal a la faringe y abarca varios procesos fisiológicos que tienen como fin cerrar el paso del contenido a la vía aérea.

- Esofágica: La relajación del músculo cricofaríngeo y el movimiento de la laringe produce la apertura del esfínter superior del esófago y una apnea momentánea, de este modo el bolo se introduce en el esófago y llega al estómago por medio de la peristalsis.

Una disfunción en cualquiera de estas fases puede conducir a la disfagia ${ }^{2,12}$. Hay 6 potenciales mecanismos por los que pacientes críticos adquieren desórdenes en la deglución, estos son: primero, traumas producidos por la intubación orotraqueal y traqueostomía, laringoscopía y mal posicionamiento de la máscara laríngea o intubación prolongada ${ }^{2,13}$. Estos pueden ser ulceraciones e inflamación en las estructuras anatómicas relacionadas con la deglución, conduciendo a una deglución inefectiva por granulación del tejido, dislocación y subluxación aritenoidea y compresión del nervio lingual además, la compresión del nervio laríngeo recurrente conlleva una paresis y parálisis de las cuerdas vocales ${ }^{2,9}$.

El segundo mecanismo consiste en la debilitación de los músculos de la deglución por causado por neuromiopatías, como la polineuromiopatía, o atrofia por falta de uso de ciertos grupos musculares ${ }^{2,5}$. El tercer y cuarto mecanismo están dados por el deterioro de la sensibilidad orofaríngea y larínea, así como del sensorio, pudiendo ser causado por delirio adquirido en la $\mathrm{UCI}$, edema local, polineuropatías, la patología clínica subyacente o los efectos de fármacos sedantes ${ }^{2}$.

El quinto mecanismo es el reflujo gastroesofágico que se origina como consecuencia de un descenso de la presión en el esfínter esofágico inferior en los pacientes críticos, estando relacionado a presión o tos superpuestos, un aumento de la presión abdominal 
- la administración de medicamentos que relajan este esfínter ${ }^{2,14}$. Todo lo anterior, sumado a la presencia de intubación endotraqueal, la posición supina que toman los pacientes en la $\mathrm{UCl}$, fármacos que retardan el vaciamiento gástrico, compromiso de la peristalsis esofágica y los bajos niveles de secreción salival; contribuyen a un aumento del reflujo gastroesofágico transitorio, inicialmente normal ${ }^{14}$. Por último, el sexto mecanismo consiste en desincronización entre la respiración y deglución en pacientes con dificultad respiratoria y taquipnea, esto se debe a un acortamiento del período apneico durante la fase esofágica de la deglución, permitiendo el paso del bolo a la vía respiratoria ${ }^{2}$.

\section{Disfagia post-extubación}

La disfagia post extubación es resultado de mecanismos varios, tanto mecánicos como cognitivos, los primeros relacionados al tubo endotraqueal y las lesiones producidas por este, mientras que los segundos son inherentes al daño cerebral por trauma o la misma patología crítica ${ }^{1,4,9}$. Macht et al., en un estudio de cohorte, observaron que la duración de la ventilación mecánica tenía una relación directa con el desarrollo de disfagia post-extubación severa. Esto, a su vez, se asocia a un peor pronóstico, estancias intahospitalarias mayores, reducción de la ingesta, colocación de tubos de alimentación y mayor duración de la hospitalización después del diagnóstico de disfagia ${ }^{1}$.

\section{Tratamiento}

El tratamiento para la disfagia presentada en el paciente crítico de cualquier etiología, no está establecido ${ }^{15}$, sin embargo, existen una serie de medidas versátiles que permitirán un aumento en la calidad de vida del paciente, las cuales se centran en la modificación de la textura de la dieta, cambios posturales, maniobras compensatorias, ejercicios terapéuticos y estimulación nerviosa ${ }^{6}$.

\section{Modificación de la textura dietética}

Modificar la textura de la dieta puede reducir el riesgo de aspiración en pa- cientes críticos, la cual es causada principalmente por la ingesta de líquidos, debido a su rápido viaje hacia la orofaringe ${ }^{16}$. El uso de espesantes comerciales y cortar los alimentos sólidos en trozos más pequeños y digeribles ayudan a disminuir drásticamente el riesgo de aspiración y sus consecuencias nocivas ${ }^{6}$.

La dieta apropiada para el paciente, debe ser provista por el médico encargado del caso en colaboración con los patólogos del habla y del lenguaje, adecuándose lo mejor posible a las preferencias dietéticas del paciente, además del riesgo/beneficio que conlleva para este ${ }^{15}$.

\section{Cambios posturales, maniobras compensatorias y Ejercicios Tera- péuticos}

Dentro de las medidas utilizadas para tratar la disfagia, podemos encontrar una serie de cambios y maniobras que van destinados, al igual que la modificación de la textura dietética, a disminuir el riesgo de la aspiración como complicación. Entre los cambios posturales se encuentran: bajar la barbilla o ladear la cabeza hacia un lado en el momento de la deglución ${ }^{15}$ ). Estos cambios deben ir acompañados de maniobras compensatorias como los intentos del paciente por tragar pequeñas cantidades, tragar varias veces, toser deliberadamente cada vez después de tragar o mantener la laringe en una posición superior con la mano en el momento de la deglución (maniobra de Mendelsohn). De forma complementaria, se puede implementar el uso de ejercicios terapéuticos, que buscan el fortalecimiento y maduración de los músculos de la deglución. Aunque la eficacia de los ejercicios terapéuticos aún es incierta ${ }^{6}$.

\section{Estimulación eléctrica Faríngea (PES)}

Consiste en la administración de una corriente eléctrica por medio de un dispositivo, que mejora la reorganización cortical y permite restaurar o mejorar la deglución en lesiones cerebrales ${ }^{6}$. Además de aumentar los niveles del neuropéptido SP el cual se ha visto relacionado con mejoras en la deglución y el reflejo de la tos en la musculatura orofaringeo, fortaleciendo los músculos que se encuentren atrofiados y proporciona retroalimentación sensorial a los centros de deglución tanto corticales como subcorticales. No obstante, no se considera útil en músculos denervados. Esta técnica aún no es completamente aceptada por los patólogos del habla y lenguaje, debido a su poco uso $y$ falta de estudio ${ }^{17}$.

\section{Complicaciones}

La disfagia producida por algunos de los procedimientos realizados en el paciente critico o como consecuencia de algunas lesiones neurológicas, puede conllevar al desarrollo de dificultades que afectan la calidad de vida del paciente. Entre las posibles complicaciones se encuentran: La aspiración, penetración, afecciones en los patrones de deglución secuencial (principalmente en pacientes con accidente cerebrovascular), molestias en la ingesta, pérdida de apetito, deshidratación, uso de una sonda nasogástrica para la alimentación y la muerte ${ }^{6,18}$.

En pacientes observados tiempo después de su recuperación, se presentaron complicaciones como temor al comer, dificultades y dolor en la masticación, la formación de un tumor primario localizado en la orofaringe, dolor al deglutir, dificultad con la ingesta oral y requerimiento de una sonda para la alimentación, lo cual podía llegar a producirles neumonía u otras afecciones pulmonares ${ }^{19}$.

\section{Aspiración y/o penetración}

La aspiración y la penetración son las complicaciones más comunes de la disfagia, presentándose en aproximadamente $44 \%$ de los pacientes recién extubados. Pueden ser causadas debido a la alta ingesta de líquidos que se presenta en muchos pacientes luego de determinados procedimientos como la traqueostomía, intubación y otros similares ${ }^{6}$. También se ha relacionado con la duración del tiempo de intubación que tenga el paciente, evidenciándose un claro aumento de la probabilidad de presentar aspiración o penetración luego de más de 10 días tras la intubación ${ }^{20}$.

También se ha demostrado que en pacientes con disfagia, producto de una 
sepsis, es más probable la aparición de aspiración o penetración, e incluso un aumento en la tasa de mortalidad pasados 4 meses, en comparación a los pacientes cuya disfagia se deba a otro motivo ${ }^{18}$. Se ha considerado que el balón de la cánula de la traqueostomía podría ser un factor protector frente a la aspiración, ya que evita el paso de secreciones a la vía aérea ${ }^{21}$.

\section{Neumonía}

La aspiración también se ha visto asociada con el desarrollo de neumonía, independientemente de la presencia o ausencia de tos en la aspiración. Aunque no se han descrito completamente los mecanismos de la neumonía por aspiración, se cree que es causada por la presencia de contenido gástrico en las vías respiratorias tras la aspiración de alimentos. Además, se encontró que en los pacientes críticos, hay una alteración en la flora bucal en la que se encuentran bacterias gramnegativas más patógenas ${ }^{2,6}$.

\section{Conclusión}

La disfagia es una complicación importante dentro de los pacientes neurocríticos, tanto por su morbilidad dentro de las instalaciones de cuidado crítico, como por las posibles comorbilidades en pa- cientes con esta afección, siendo estos propensos a infecciones respiratorias, pérdida del apetito, dolor y molestias al tragar, disfonía, deshidratación y falla respiratoria, entre otras. Considerando esto, es importante recurrir a las medidas terapéuticas con el fin de reducir la probabilidad de una patología o complicación secundaria a la disfagia que pueda comprometer la calidad de vida, o la vida misma, del paciente. Los neurocirujanos debemos recocer esta condición en nuestros pacientes para aportar al manejo integral de nuestros pacientes.

Recibido: 19 de diciembre de 2018 Aceptado: 28 de diciembre de 2018

\section{Referencias}

1. Macht M, Wimbish T, Clark BJ, Benson AB, Burnham EL, Williams A, et al. Postextubation dysphagia is persistent and associated with poor outcomes in survivors of critical illness. Crit Care. 2011;15(5):R231.

2. Macht M, Wimbish T, Bodine C, Moss M. ICU-acquired swallowing disorders. Crit Care Med. 2013;41(10):2396-405.

3. Skoretz SA, Flowers HL, Martino R. The incidence of dysphagia following endotracheal intubation a systematic review. Chest. 2010;137(3):665-73.

4. Kwok AM, Davis JW, Cagle KM, Sue LP, Kaups KL. Post-extubation dysphagia in trauma patients: It's hard to swallow. Am J Surg. 2013;206(6):924-8.

5. Aydogdu I, Kiylioglu N, Tarlaci S, Tanriverdi Z, Alpaydin S, Acarer A, et al. Clinical Neurophysiology Diagnostic value of " dysphagia limit "' for neurogenic dysphagia : 17 years of experience in 1278 adults. 2015;126:634-43.

6. Macht M, White SD, Moss M. Swallowing Dysfunction After Critical Illness. Chest. 2014;146(6):1681-9.

7. Fernández Carmona A, Macías Guarasa I, Gutiérrez Rodríguez R, Martínez López P, Díaz Castellanos M. Dysphagia Following Prolonged Mechanical Ventilation and Tracheostomy in Critical III Patients. Results of Edisval Study Pilot Phase. Intensive Care Med Exp. 2015;3(Suppl 1):A677.

8. Kothari M, Bjerrum K, Nielsen LH, Jensen J, Nielsen JF. Influence of External Subglottic Air Flow on Dysphagic Tracheotomized Patients with Severe Brain Injury: Preliminary Findings. Ann Otol Rhinol Laryngol. 2017;126(3):199-204.

9. Oliveira ACM de, Friche AA de L, Salomão MS, Bougo GC, Vicente LCC. Predictive factors for oropharyngeal dysphagia after prolonged orotracheal intubation. Braz J Otorhinolaryngol. 2017;(xx).

10. Behera A, Read D, Jackson N, Saour B, Alshekhlee D, Mosier AK. A Validated Swallow Screener for Dysphagia and Aspiration in Patients with Stroke. J Stroke Cerebrovasc Dis. 2018;27(7):1897-904.

11. Moraes DP, Sassi FC, Mangilli LD, Zilberstein B, de Andrade CRF. Clinical prognostic indicators of dysphagia following prolonged orotracheal intubation in ICU patients. Crit Care. 2013;17(5):1-10.

12. Lancaster J. Dysphagia: its nature, assessment and management. Br J Community Nurs. 2015;20(Sup6a):S28-32.

13. Anil Kumar V, Ushashree Reddy B, Kiran Kumar VA, Pal R, Kumar L, Jahnavi M, et al. Speech and Swallowing Function Outcome Following Early Tracheostomy in Patients Who Underwent Neurosurgical Intervention.

14. Aderinto-Adike AO, Quigley EMM. Gastrointestinal motility problems in critical care: A clinical perspective. J Dig Dis. 2014;15(7):335-44.

15. Macht M, Wimbish T, Clark BJ, Benson AB, Burnham EL, Williams A, et al. Diagnosis and treatment of post-extubation dysphagia: Results from a national survey. J Crit Care. 2012 Dec;27(6):578-86.

16. Via MA, Mechanick JI. Malnutrition, Dehydration, and Ancillary Feeding Options in Dysphagia Patients. Otolaryngol Clin North Am. 2013 Dec;46(6):1059-71.

17. Muhle P, Suntrup-Krueger S, Bittner S, Ruck T, Claus I, Marian T, et al. PB3. Pharyngeal Electrical Stimulation in neurointensive care patients suffering from severe post-stroke dysphagia - Post stimulation increase of salivary substance $P$ level may indicate treatment success. Clin Neurophysiol. 2018;129(8):e55-6.

18. Zielske J, Bohne S, Brunkhorst FM, Axer H, Guntinas-Lichius O. Acute and long-term dysphagia in critically ill patients with severe sepsis: results of a prospective controlled observational study. Eur Arch Oto-Rhino-Laryngology. 2014;271(11):3085-93.

19. Kraaijenga SAC, Oskam IM, van der Molen L, Hamming-Vrieze O, Hilgers FJM, van den Brekel MWM. Evaluation of long term (10-years+) dysphagia and trismus in patients treated with concurrent chemo-radiotherapy for advanced head and neck cancer. Oral Oncol. 2015 Aug;51(8):787-94.

20. Scheel R, Pisegna JM, McNally E, Noordzij JP, Langmore SE. Endoscopic Assessment of Swallowing after Prolonged Intubation in the ICU Setting. Ann Otol Rhinol Laryngol. 2016;125(1):43-52.

21. Alvo A, Olavarría C. Decannulation and Assessment of Deglutition in the Tracheostomized Patient in Non-Neurocritical Intensive Care. Acta Otorrinolaringol (English Ed. 2014;65(2):114-9.

Correspondencia a:

Dr. Luis Rafael Moscote-Salazar

rafaelmoscote21@gmail.com 